\title{
FOTODEGRADAÇÃO DE POLÍMEROS CLORADOS IRRADIADOS COM A LUZ SÍNCROTRON - I. EMISSÃO DE GASES
}

Claudia Santos Cardoso de Castro e Gerardo Gerson Bezerra de Souza

Instituto de Química, Departamento de Físico-Química, Universidade Federal do Rio de Janeiro, 21949-900 Rio de Janeiro - RJ Elisabeth Ermel da Costa Monteiro

Instituto de Macromoléculas, Universidade Federal do Rio de Janeiro, 21945-970 Rio de Janeiro - RJ

Recebido em 3/1/00; aceito em 26/10/00

\begin{abstract}
PHOTODEGRADATION OF CHLORINATED POLYMERS IRRADIATED WITH SYNCHROTRON LIGHT. Chlorinated polymers (PVC, PVDC and E-CTFE) were irradiated with white light produced at the Brazilian Synchrotron Light Source (LNLS). The emitted gases were analyzed by mass spectrometry. The spectra were dominated by peaks related to hydrochloric acid, $\mathrm{HCl}$, and chlorine $\left({ }^{35} \mathrm{Cl}\right)$. The measured $\mathrm{HCl}$ intensity is used to evaluate the sensitivity of the polymers over a broad energy range. PVDC showed the greatest light sensitivity as compared to PVC and E-CTFE.
\end{abstract}

Keywords: polymers; photodegradation; synchrotron radiation.

\section{INTRODUÇÃO}

O estudo de reações fotoquímicas em polímeros ainda hoje se constitui uma área de intensa investigação científica. Entre os fatores que contribuem para a importância desse estudo, podem-se citar os seguintes: a compreensão dos processos fotoquímicos em polímeros desempenha papel fundamental na construção de microdispositivos eletrônicos e mecânicos, como por exemplo na técnica de LIGA $^{1}$; polímeros são materiais amplamente empregados na embalagem de produtos industriais e o conhecimento dos processos de desgaste e de envelhecimento devido à exposição à luz é de extrema importância para a indústria ${ }^{2}$. Apesar de sua relevância, os mecanismos de degradação induzidos por fótons em polímeros, que desencadeiam modificações em suas propriedades físicas e químicas, ainda não estão completamente elucidados ${ }^{3}$.

Muitos polímeros, ao serem irradiados com fótons de alta energia, emitem gases. Considerando os diversos processos fotoquímicos, uma das maneiras de fazer a caracterização das modificações estruturais nos polímeros é, conseqüentemente, analisar os gases emitidos durante a irradiação. Os produtos voláteis emitidos por poli (cloreto de vinila) (PVC) sob irradiação UV já foram estudados ${ }^{4,5}$, mostrando que, por exemplo, existe uma intensa emissão de $\mathrm{HCl}$. A análise de gases produzidos por um número grande de materiais poliméricos sob irradiação gama também já foi efetuada ${ }^{6}$. Em todos os casos, foi observada emissão gasosa em conseqüência da exposição a fótons energéticos.

No entanto, os mecanismos químicos que levam à formação de tais gases são de extrema complexidade. No caso do PVC, um dos materiais poliméricos mais estudados, existem evidências bastante fortes de que o principal tipo de degradação da cadeia polimérica, independente da fonte de excitação (se térmica, termo-oxidante ou por luz), é a eliminação em seqüência de $\mathrm{HCl}$ e a formação simultânea de ligações duplas conjugadas (cadeias poliênicas) $^{7}$. O mecanismo que desencadeia tal reação em cascata ainda não está bem esclarecido. A liberação de $\mathrm{HCl}$, que é um gás altamente corrosivo, tem, portanto, como conseqüência, a formação de cadeias poliméricas de alto grau de reatividade e que absorvem fortemente na região do visível até UV.

No presente trabalho, foram realizadas medidas da intensidade de emissão de gases por um conjunto de polímeros contendo cloro, induzida pela luz síncrotron (luz branca). A identificação dos fragmentos produzidos foi feita através da técnica de espectrometria de massas. Como fonte de excitação, foi utilizada a radiação produzida no Laboratório Nacional de Luz Síncrotron (LNLS), em Campinas, São Paulo. O principal objetivo deste estudo é compreender os mecanismos de degradação de polímeros induzidos pela luz em função da dose de exposição. Os seguintes polímeros foram analisados: poli (cloreto de vinila) (PVC), poli (cloreto de vinilideno) (PVDC) e co-polímero de etileno-cloro-tri-fluoroetileno (E-CTFE). O grau de degradação relativo foi analisado pela medida de $\mathrm{HCl}$ liberado em cada caso.

\section{PARTE EXPERIMENTAL}

As medidas foram realizadas no Laboratório Nacional de Luz Síncrotron (LNLS), utilizando-se a linha de luz TGM ("Toroidal Grating Monochromator"). Esta linha possui um monocromador capaz de selecionar fótons de energia na faixa de 12 a $320 \mathrm{eV}^{8}$. No entanto, por questões de intensidade de luz, as amostras foram irradiadas utilizando a ordem zero, isto é, foram expostas a fótons com energia variando entre o visível e os raios-X. Grande parte dos fótons produzidos pelo LNLS se encontra na faixa do ultravioleta de vácuo e raios-X. Energias inferiores a $20 \mathrm{eV}$ correspondem a aproximadamente $0,2 \%$ do total da intensidade de emissão de fótons. A descrição da linha TGM está detalhada em outra publicação ${ }^{8}$.

A intensidade do feixe de luz síncrotron nas amostras foi monitorada no decorrer das experiências por um fotodiodo do tipo Schottky (modelo Hamamatsu G1127-02), cuja sensibilidade está restrita à região do UV próximo até raios-X próximos.

$\mathrm{O}$ sistema de medida foi instalado numa câmara de ultraalto vácuo, com uma bomba turbo-molecular, atingindo-se uma pressão de base da ordem de $1 \times 10^{-9}$ mbar. Todas as medidas foram realizadas à temperatura ambiente, sob pressões que variaram entre $5 \times 10^{-9}$ mbar a $2 \times 10^{-7}$ mbar.

As amostras de PVC foram preparadas pela técnica de "spin casting", enquanto as de PVDC e E-CTFE foram adquiridas comercialmente (Goodfellow Cambridge Ltd.). As respectivas espessuras dos filmes utilizados foram $20 \mu \mathrm{m}, 33 \mu \mathrm{m}$, and 12,5 $\mu \mathrm{m}$. As amostras não foram sujeitas a qualquer procedimento adicional de limpeza, tendo sido irradiadas como foram recebidas.

A análise dos gases emitidos foi realizada através de um espectrômetro de massas comercial, do tipo quadrupolo, modelo SRS200 (Stanford Research Systems), capaz de medir massas 
na faixa de 1 a 200 u.m.a.. A fonte ionizante do quadrupolo foi posicionada a uma distância em torno de $20 \mathrm{~cm}$ da superfície da amostra. A troca de amostras foi realizada sem quebra de vácuo, utilizando-se um manipulador de alta precisão. Deste modo, todas as amostras foram irradiadas sob as mesmas condições experimentais. $\mathrm{O}$ fotodiodo para controle da intensidade do feixe foi colocado no mesmo suporte das amostras. Sendo assim, tanto as amostras como o fotodiodo ficaram expostos ao mesmo ângulo sólido de incidência da radiação.

Os espectros de massas foram obtidos medindo-se a intensidade de cada fragmento iônico num intervalo pré-selecionado. Foi utilizado um copo de Faraday como instrumento de detecção. Pressões parciais de gases na câmara de análise inferiores a $1 \times 10^{-9}$ mbar são dificilmente detectadas. A medida de cada espectro foi realizada num ponto virgem da amostra.

Para a variação da dose ministrada às amostras, utilizou-se um par de fendas posicionado ao longo da linha de luz. O tamanho da abertura das fendas foi controlado por um micrômetro, com variação entre 0 e $2 \mathrm{~mm}$.

Uma inspeção visual da superfície das amostras mostrou que os pontos irradiados apresentavam modificação de cor, indo do transparente característico da região não irradiada para uma coloração amarelada, podendo chegar até um marrom opaco, dependendo da dose de exposição (quanto maior a dose, mais escura se tornava a amostra). A observação das regiões irradiadas mostrou que o feixe incidente sobre a superfície do polímero tinha dimensões da ordem de $1 \mathrm{~mm}^{2}$.

\section{RESULTADOS}

As Figuras 1, 2 e 3 mostram, respectivamente, os espectros de massa obtidos para as amostras de PVDC, PVC e E-CTFE, cobrindo a faixa de massa de 1 a 50 u.m.a.. A contribuição devida aos gases residuais presentes no sistema (fundo) foi anteriormente subtraída. Não foram observados picos na região do espectro de massa superior a 50 u.m.a.

Nota-se que, em todos os casos, a emissão mais intensa ocorre para o fragmento de massa 36 , correspondente ao $\mathrm{HCl}$, contendo o isótopo ${ }^{35} \mathrm{Cl}$. Observa-se também a presença da massa 38 , correspondente ao $\mathrm{HCl}$ composto pelo isótopo ${ }^{37} \mathrm{Cl}$. Existe ainda uma pequena quantidade de ${ }^{35} \mathrm{Cl}$ liberada pelos processos de fotodecomposição. Devido à pequena abundância isotópica de ${ }^{37} \mathrm{Cl}$, não foi possível medilo, considerando a sensibilidade do espectrômetro utilizado. Os espectros de gases residuais do sistema, na faixa de massa de 35 a 38 , não apresentaram picos. Este fato demonstra inequivocamente a ocorrência de processos de degradação nos polímeros expostos à luz branca. A emissão mais intensa de $\mathrm{HCl}$ está de acordo com resultados já obtidos na literatura ${ }^{4}$.

Os espectros de massa de PVC e E-CTFE (Figuras 2 e 3) mostram também a emissão de $\mathrm{H}_{2}$. Este gás pode ter tido origem no próprio polímero, devido aos processos de recombinação na liberação de gases, ou pode ter surgido em decorrência da presença de alguma impureza existente na superfície do polímero.

No caso particular de E-CTFE, observa-se que o espectro é mais rico em picos, sendo possível identificar um fragmento de relação carga/massa igual a 20 e que pode ser associado à formação de ${ }^{1} \mathrm{H}^{19} \mathrm{~F}$, demonstrando uma vez mais a existência de processos de fotodegradação. Nota-se além disso a presença dos fragmentos de massas 17 e 18 , correpondentes respectivamente a $\mathrm{OH}$ e $\mathrm{H}_{2} \mathrm{O}$, que são representativos da existência de água. Devido aos processos de interação do polímero com a radiação, a superfície pode ter ficado susceptível à adsorção de água. A presença de $\mathrm{H}_{2} \mathrm{O}$ na câmara de análise foi constatada pela presença nos espectros de gases residuais do sistema (obtidos sem feixe de luz) de dois picos nas posições correspondentes às massas 17 e 18 .

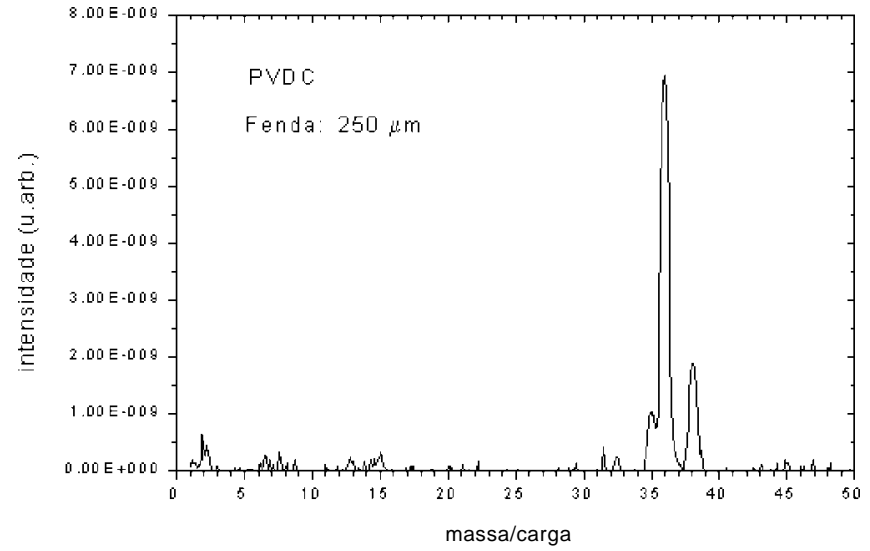

Figura 1. Emissão de gases por PVDC irradiado com luz síncrotron. As massas 35, 36 e 38 correspondem respectivamente $a{ }^{35} \mathrm{Cl},{ }^{1} \mathrm{H}^{35} \mathrm{Cl}$ $e^{1} \mathrm{H}^{37} \mathrm{Cl}$.

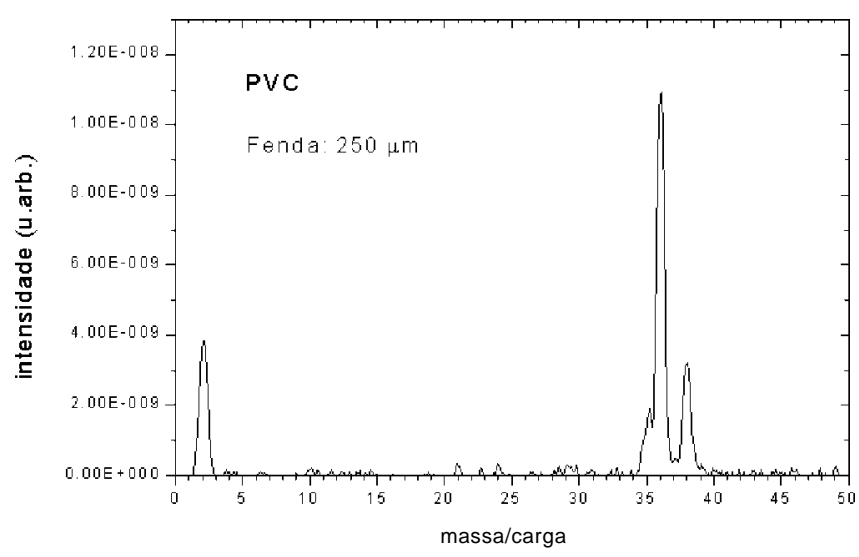

Figura 2. Emissão de gases por PVC irradiado com luz síncrotron. As massas 2, 35, 36 e 38 correspondem respectivamente $a{ }^{l} \mathrm{H}_{2},{ }^{35} \mathrm{Cl}$, ${ }^{1} \mathrm{H}^{35} \mathrm{Cl} \mathrm{e}^{1} \mathrm{H}^{37} \mathrm{Cl}$

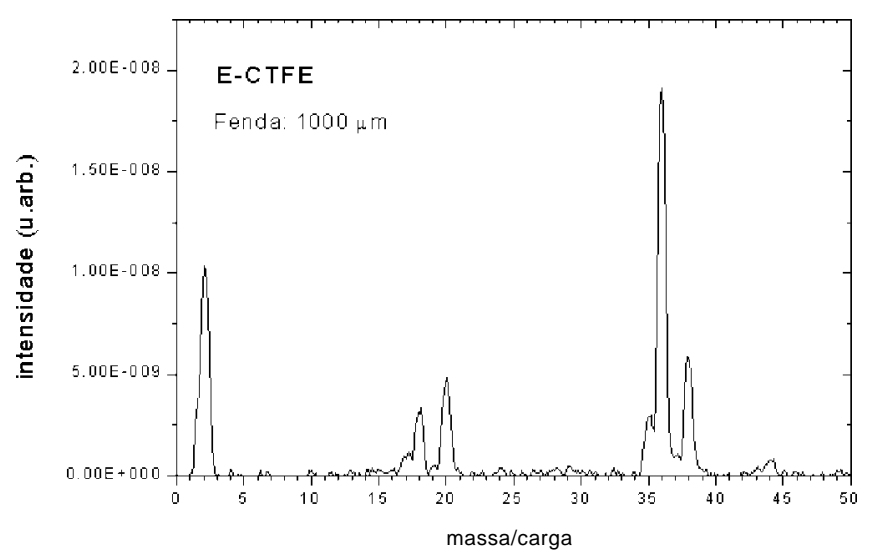

Figura 3. Emissão de gases por E-CTFE irradiado com luz síncrotron. As massas 1, 17, 18, 20, 35, 36 e 38 correspondem respectivamente $a^{1} \mathrm{H}_{2},{ }^{16} \mathrm{O}^{1} \mathrm{H},{ }^{1} \mathrm{H}_{2}{ }^{16} \mathrm{O},{ }^{1} \mathrm{H}^{19} \mathrm{~F},{ }^{35} \mathrm{Cl},{ }^{1} \mathrm{H}^{35} \mathrm{Cl} e{ }^{1} \mathrm{H}^{37} \mathrm{Cl}$.

Outro ponto de relevância, que pode ser observado nas Figuras 4,5 e 6 , é o aumento na quantidade da emissão de alguns fragmentos com a dose. Em particular, nota-se o crescimento de $\mathrm{HCl}$ emitido em todos os polímeros investigados no presente trabalho.

A modificação de cor dos polímeros na região irradiada é um indício de que houve formação de ligações duplas conjugadas em conseqüência da exposição à luz, o que está de acordo com os resultados da literatura ${ }^{7}$. 


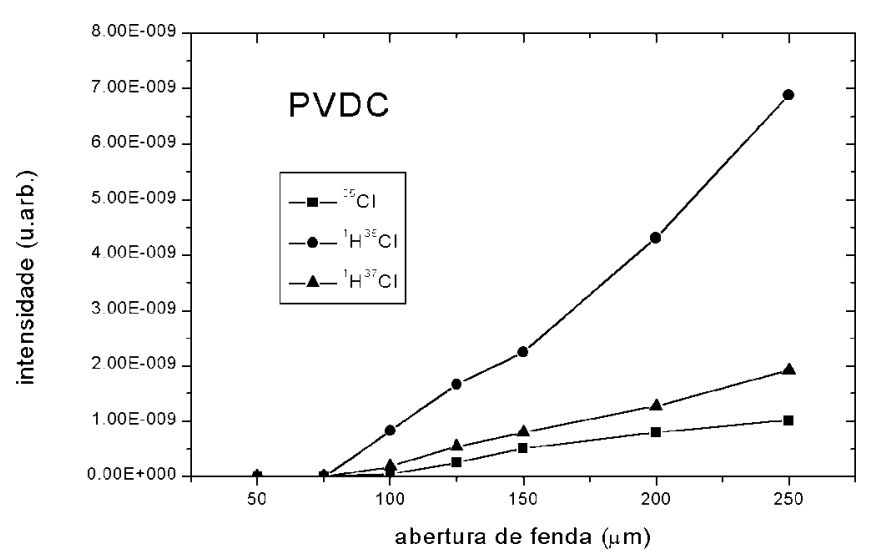

Figura 4. Taxa de emissão de gases por PVDC durante a exposição à luz síncrotron.

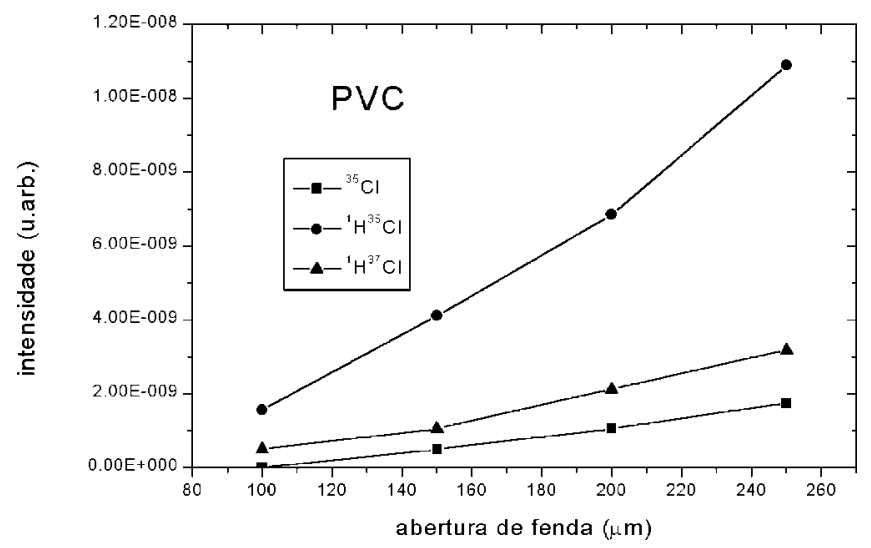

Figura 5. Taxa de emissão de gases por PVC durante a exposição à luz síncrotron.

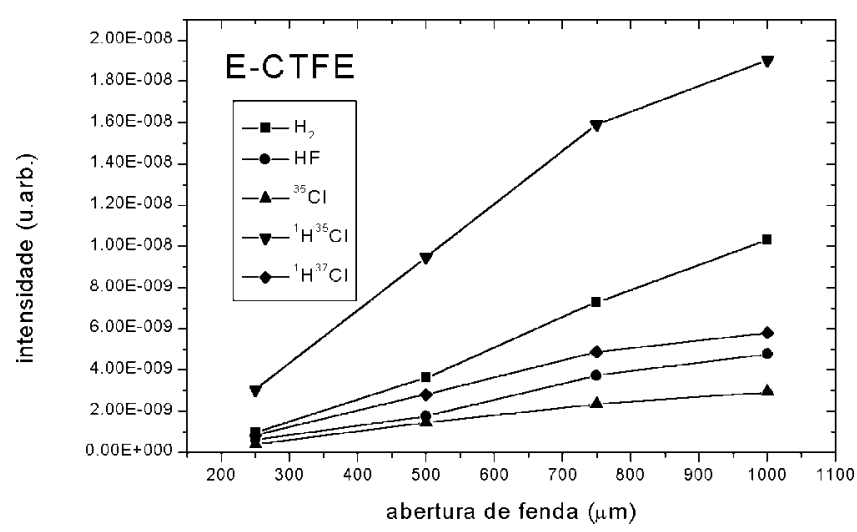

Figura 6. Taxa de emissão de gases por E-CTFE durante a exposição à luz síncrotron.

\section{DISCUSSÃO}

\section{Fluxo de Fótons}

A Figura 7 mostra o comportamento da corrente no fotodiodo em função da abertura das fendas. Os valores apresentados estão normalizados a $100 \mathrm{~mA}$, que é a corrente média do feixe de elétrons no anel do LNLS. Comparam-se na mesma figura os valores experimentais com um ajuste linear e com um polinomial de grau 4. A função polinomial obteve melhores resultados para o ajuste, indicando que o perfil do feixe de luz incidente sobre a amostra não é uniforme, podendo ter uma forma aproximadamente gaussiana ${ }^{9}$.

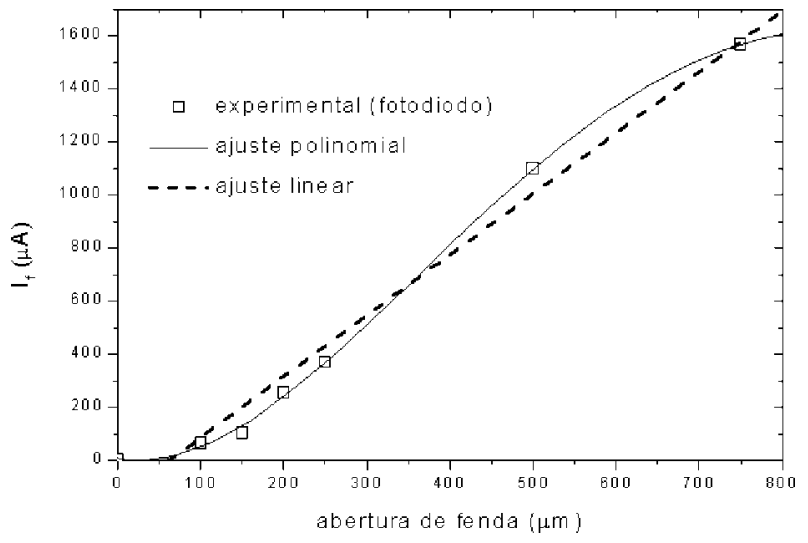

Figura 7. Corrente no fotodiodo normalizada à corrente de $100 \mathrm{~mA}$ do feixe de elétrons do LNLS.

\section{Sensibilidade dos polímeros à fotodegradação}

As Figuras 4, 5, 6 mostram a evolução da intensidade de emissão de alguns gases em função da abertura de fendas, evidenciando seu crescimento em função da dose de exposição. Embora as amostras tenham sido expostas à luz branca, é provável que fótons na faixa de energia do ultra-violeta sejam a principal fonte de excitação do polímero. Este resultado foi encontrado por Andrady et al. ${ }^{2}$, os quais verificaram que o máximo de dano causado numa série de polímeros ocorria na faixa do UV com pequenos comprimentos de onda (UV-B). Fótons na faixa do visível não têm energia suficiente para excitar elétrons na banda de valência do material. Ainda assim, uma análise mais profunda do efeito da luz branca na fotodegradação requer mais investigações.

Além de ser comum aos polímeros analisados neste trabalho, $\mathrm{HCl}$ foi o produto volátil mais intensamente emitido em todos os casos. Por ser um gás característico dos processos de fotodegradação ${ }^{7}$, ele será usado a seguir como indicador ao fazermos uma análise de sensibilidade dos polímeros à luz branca.

A Figura 8 mostra a intensidade de emissão de $\mathrm{HCl}$ para PVC, PVDC e E-CTFE em função da corrente do fotodiodo $\left(\mathrm{I}_{\mathrm{di}}\right)$ normalizada pela corrente de elétrons do anel do síncrotron $\left(\mathrm{I}_{\mathrm{el}}\right)$ no instante da medida. A corrente de elétrons no anel diminui com o tempo. Nota-se que a tendência de crescimento da intensidade de emissão de $\mathrm{HCl}$ é diferente para cada polímero analisado. A maior taxa de crescimento é a do PVDC e a menor é a do E-CTFE. Dentro do critério estabelecido, podemos inferir que o PVDC é o polímero mais sensível à radiação branca em comparação com PVC e E-CTFE. Pode-se ajustar uma reta aos dados da Figura 8 para avaliar a taxa de crescimento em cada caso. Os valores das inclinações das retas para as intensidades de PVDC, PVC e E-CTFE na Figura 8 são respectivamente $3,6 \times 10^{-6}, 1,5 \times$ $10^{-6}$ e $0,3 \times 10^{-6}$. Como é amplamente conhecido, o monômero do PVDC contém duas vezes mais ligações $\mathrm{C}-\mathrm{Cl}$ do que o PVC. A taxa de crescimento da emissão de $\mathrm{HCl}$ no PVDC é aproximadamente o dobro da de PVC, conforme mostrado na Figura 8. Este fato pode indicar que os processos de degradação para formação de $\mathrm{HCl}$ dependem do número de ligações $\mathrm{C}-\mathrm{Cl}$, como esperado. Comparando-se a seguir com o E-CTFE, observa-se que este último apresenta uma taxa crescimento da intensidade de $\mathrm{HCl}$ uma ordem de grandeza inferior ao PVDC. Pode-se supor portanto, baseando-se no raciocínio anterior, que o número de ligações $\mathrm{C}-\mathrm{Cl}$ seja aproximadamente 10 vezes menor no co-polímero em relação ao PVDC. Sabe-se que o teor de etileno no E-CTFE pode variar $^{10}$, variando conseqüentemente o número de ligações $\mathrm{C}-\mathrm{Cl}$ presentes no co-polímero. 


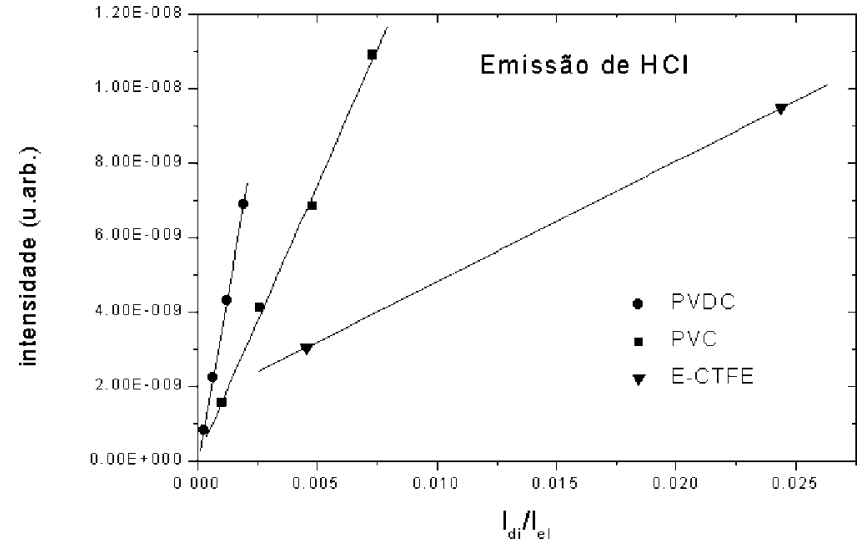

Figura 8. Comparação entre as intensidades de emissão de ${ }^{1} \mathrm{H}^{35} \mathrm{Cl}$ para PVC, PVDC e E-CTFE. $I_{d i}$ e $I_{e l}$ representam respectivamente a corrente do fotodiodo e a corrente de elétrons no anel do síncrotron. As retas correspondem ao ajuste linear.

Partindo do princípio que a formação de $\mathrm{HCl}$ depende do número de ligações $\mathrm{C}-\mathrm{Cl}$ nos polímeros, pode-se compreender a presença de $\mathrm{H}_{2}$ nos espectros de PVC e E-CTFE (Figuras 2 e 3). As ligações C-H também são quebradas nos processos de fotodegradação e os radicais hidrogênio liberados, devido à interação da luz com o polímero, se recombinam, formando $\mathrm{H}_{2}$. No caso particular do E-CTFE, a recombinação também pode ser feita com o flúor gerando HF (massa 20). Na Figura 3, observa-se a presença de HF, confirmando a ocorrência desse tipo de reação.

\section{CONCLUSÃO}

A análise de gases emitidos por polímeros irradiados por luz síncroton branca fornece informações relevantes acerca dos mecanismos de degradação pela interação de fótons com estes materiais. Mais especificamente, em polímeros contendo $\mathrm{Cl}$, a formação de $\mathrm{HCl}$ indica a ocorrência de processos de fotodegradação, uma vez que ligações C-Cl são quebradas, dando origem a ligações duplas conjugadas. Os polímeros investigados neste trabalho, PVC, PVDC e E-CTFE, apresentaram intensa emissão de $\mathrm{HCl}$ quando expostos à radiação branca produzida no Laboratório Nacional de Luz Síncrotron, na linha TGM.

A medida da intensidade de $\mathrm{HCl}$, por ser uma impressão digital da ocorrência de reações fotoquímicas, pode ser usada como uma forma de aferir a sensibilidade de polímeros que contêm $\mathrm{Cl}$ quando expostos a fótons de amplo espectro de energia.

\section{AGRADECIMENTOS}

Os autores agradecem à equipe da linha TGM do LNLS pelo suporte durante as experiências. $\mathrm{O}$ apoio financeiro do LNLS, UFRJ, FAPERJ e CNPq foram de enorme importância para a realização deste trabalho.

\section{REFERÊNCIAS}

1. Wollersheim, O.; Zumaqué, H.; Hormes, J.; Kadereit, D.; Langen, J.; Häußling, L.; Hoessel, P.; Hoffmann, G.; Nucl. Instrum. and Meth. in Phys. Res. B. 1995, 97, 273.

2. Andrady, A. L.; Amin, M. B.; Hamid, S. H.; Hu, X.; Torikai, A.; Ambio. 1995, 24, 191.

3. Simons, J. K.; Frigo, S. P.; Taylor, J. W.; Rosenberg, R. A.; J. Vac. Sci. Technol. A. 1994, 12, 681.

4. Straus, R. R.; Tromberg, S.; Achhammer, B. G.; J. Polym. Sci. 1959, 35, 355.Kasatotschkin, W. I.; Berlin, A. A.; Izv. AN SSSR, Ser. Kchim. 1965, 1003.

5. Carlsson, D. J.; Krzymien, M.; Worsfold, D. J.; Day, M.; J. Vinyl \& Add. Tech. 1997, 2, 100.

6. Morgan, J. T.; Stapleton, G. B.; Gas Evolution from Plastic Materials by High Energy Radiation, Rutherford Laboratory Internal Report RL-74-021. 1974.

7. Iván, B.; Polymer Durability, Degradation, Stabilization, and Lifetime Prediction, American Chemical Society 1996.

8. Fonseca, P. de T.; Pacheco, J. G.; d'A Samogin, E.; de Castro, A. R. B.; Rev. Sci. Instrum. 1992, 63, 1256.

9. Brefeld, W., Gürtler, P., Handbook on Synchrotron Radiation, editado por Ebashi, S., Koch, M., Rubenstein, E., Elsevier Science Publishers B.V., 1991, 4, cap. 7, 287.

10. Sibilia, J. P., Roldan, L., Chandrasekaran, S., J. Polym. Sci. 1972, Part A-2, 10, 549. 